\title{
The pleckstrin homology domain of phospholipase D1 accelerates EGFR endocytosis by increasing the expression of the Rab5 effector, rabaptin-5
}

\author{
Mi Hee Park ${ }^{1}$, Kang-Yell Choi ${ }^{2,3}$ and Do Sik Min ${ }^{1,3,4}$ \\ Endocytosis is differentially regulated by hypoxia-inducible factor- $1 \alpha$ (HIF-1 $\alpha$ ) and phospholipase D (PLD). However, the \\ relationship between HIF-1 $\alpha$ and PLD in endocytosis is unknown. HIF-1 $\alpha$ is degraded through the prolyl hydroxylase (PHD)/von \\ Hippel-Lindau (VHL) ubiquitination pathway in an oxygen-dependent manner. Here, we show that PLD1 recovers the decrease in \\ epidermal growth factor receptor (EGFR) endocytosis induced by HIF-1 $\alpha$ independent of lipase activity via the Rab5-mediated \\ endosome fusion pathway. EGF-induced interaction of PLD1 with HIF-1 $\alpha$, PHD and VHL may contribute to EGFR endocytosis. \\ The pleckstrin homology domain (PH) of PLD1 itself promotes degradation of HIF-1 $\alpha$, then accelerates EGFR endocytosis via \\ upregulation of rabaptin-5 and suppresses tumor progression. These findings reveal a novel role of the PLD1-PH domain as a \\ positive regulator of endocytosis and provide a link between PLD1 and HIF-1 $\alpha$ in the EGFR endocytosis pathway. \\ Experimental \& Molecular Medicine (2015) 47, e200; doi:10.1038/emm.2015.101; published online 18 December 2015
}

\section{INTRODUCTION}

Endocytosis regulates cell signaling by controlling the number of receptors available for activation in the plasma membrane, and the activation of receptors or downstream effectors often stimulates receptor endocytosis. ${ }^{1}$ The endocytosis of many signaling receptors is stimulated by ligand-induced activation. The epidermal growth factor receptor (EGFR) is the most wellstudied receptor tyrosine kinase (RTK), and EGFR endocytosis has an important role in regulation of signaling processes such as cell proliferation, survival, migration and differentiation. Phospholipase D (PLD) is a key mediator of EGFR function that can be directly regulated by upstream binding partners in an EGF-dependent manner. ${ }^{2}$ EGF triggers the activation of PLD in various cell types. ${ }^{2}$ PLD has an important role in cancer progression. $^{3-6}$ PLD catalyzes the hydrolysis of phosphatidylcholine (PC) to generate phosphatidic acid (PA) and choline. There are two mammalian isoforms of PLD, PLD1 and PLD2, which have similar structures containing pleckstrin homology (PH) and phox homology (PX) domains. ${ }^{7}$ PLD has been reported to be involved in EGFR-mediated endocytosis and PA is involved in the membrane curvature required for vesicle budding. ${ }^{8}$ Interestingly, PLD is required for EGFR endocytosis through both guanosine triphosphatases (GTPase)-activating protein (GAP) activity of its PX domain for dynamin and the production of PA. $^{9}$ Endocytosis by both clathrin-dependent and clathrin-independent mechanisms delivers receptors to early endosomes. Specific signaling complexes can be assembled through their recruitment to the early endosomal resident protein, Rab5 GTPase, which regulates fusion of endocytic vesicles to form the early endosome and has a role in the formation of clathrin-coated vesicles at the plasma membrane. ${ }^{10}$ Tumor hypoxia, which is associated with disease progression and resistance to conventional cancer therapies, intensifies numerous RTKs-mediated signaling pathways that are critical for driving oncogenesis through largely unknown mechanims. ${ }^{11,12}$ It has been reported that hypoxia-inducible factor (HIF) decelerates EGFR endocytosis via HIF-dependent downregulation of rabaptin-5, a critical Rab5 effector, at the level of transcription, resulting in prolonged and accentuated EGFR signaling to promote proliferation and cell survival. ${ }^{13}$ The level of HIF- $1 \alpha$ is positively correlated with cancer progression and poor prognosis, suggesting a critical role of HIF in oncogenesis. ${ }^{14}$ HIF- $1 \alpha$ has a central role in cellular adaptation to changes in oxygen availability and is degraded

\footnotetext{
${ }^{1}$ Department of Molecular Biology, College of Natural Science, Pusan National University, Busan, Republic of Korea; ${ }^{2}$ Department of Biotechnology, College of Life Science and Biotechnology, Yonsei University, Seoul, Republic of Korea; ${ }^{3}$ Translational Research Center for Protein Function Control, Yonsei University, Seoul, Republic of Korea and ${ }^{4}$ Genetic Engineering Institute, Pusan National University, Busan, Republic of Korea Correspondence: Professor DS Min, Department of Molecular Biology, College of Natural Science, Pusan National University, 30 Jangjeon dong, Geumjeong gu, Busan 609-735, Republic of Korea.

E-mail: minds@pusan.ac.kr
}

Received 30 September 2015; revised 21 October 2015; accepted 23 October 2015 
through the prolyl hydroxylase (PHD) and von Hippel-Lindau (VHL) ubiquitination pathway. We recently demonstrated that PLD protein itself and its $\mathrm{PH}$ domain promotes degradation of HIF- $1 \alpha$ via interaction with HIF- $1 \alpha$, PHD and VHL. ${ }^{15}$ In this study, we demonstrate that the PH domain of PLD1 recovers the decrease in EGFR endocytosis in response to HIF- $1 \alpha$ via destabilization of HIF- $1 \alpha$ and upregulation of rabatin-5, suggesting a novel role of the PLD1 PH domain in endocytosis linked with HIF-1 $\alpha$.

\section{MATERIALS AND METHODS}

\section{Cell culture and transfection}

HEK293 and HT29 cells were cultured in Dulbecco's modified Eagle's medium with $10 \%$ heat-inactivated fetal bovine serum containing $1 \%$ antibiotic-antimycotic (Invitrogen, Carlsbad, CA, USA) at $37^{\circ} \mathrm{C}$ in a $5 \% \mathrm{CO}_{2} / 95 \%$ air incubator. Hypoxia was induced by exposing cells to $1 \% \mathrm{O}_{2} / 5 \% \mathrm{CO}_{2}$ balanced with $\mathrm{N}_{2}$ using a hypoxic chamber (Forma, Costa Mesa, CA, USA). Cells were transfected using Lipofectamine Plus or Lipofectamine 2000 (Invitrogen) according to the manufacturer's instructions. To generate PLD1-PH-overexpressing cells, HT29 cells were transfected with pGFP-PLD1-PH and selected using G418 (Sigma, St Louis, MO, USA).

\section{Materials}

EGF was purchased from Sigma. Alexa Fluor 555-EGF was obtained from Invitrogen. Cycloheximide and MG132 were purchased from AG scientific (San Diego, CA, USA).

\section{Immunoblot and immunoprecipitation}

Cells were lysed in passive-lysis buffer (Promega, Madison, WI, USA), followed by gentle sonication. Cell lysates were incubated with suitable antibody in the presence of protein A sepharose beads (Amersham, Piscataway, NJ, USA). Immunoprecipitates and cell lysates were boiled with SDS sample buffer, then separated by SDS-polyacrylamide gel electrophoresis. The antibodies used in immunoblot and immunoprecipitation were: EGFR, HIF-1 $\alpha, \alpha$-tubulin, green fluorescent protein (GFP) and hemagglutanin (Santa Cruz Biotechnology, Santa Cruz, CA, USA); p-ERK, ERK and hydroxylated-HIF-1 $\alpha$ (Cell Signaling Technologies, Beverly, MA, USA); HIF-1 $\alpha$ (BD Bioscience, Rockville, MD, USA). A polyclonal anti-PLD antibody that recognizes both PLD1 and PLD2 was generated as previously described. ${ }^{16}$

\section{Fluorescence microscopy}

HEK293 cells were transfected with fluorescent-fusion plasmid and various constructs, after which live cells were visualized and images were collected using a fluorescence microscope (Axiovert 200M, Zwiss, Jena, Germany). Cells were visualized and images were collected using a fluorescence microscope. The percentage of GFP-Rab5activation cells was calculated based on the total GFP-positive cells that displayed at least one enlarged endosome. At least 300 cells were scored for enlarged endosomes per experiment. The results are expressed as the mean \pm s.d. based on three independent experiments. HEK293 cells were treated with Alexa Fluor 555-EGF for the indicated time, then washed in ice-cold phosphate-buffered saline and fixed in $4 \%$ paraformaldehyde for 10 min. Cells were visualized and images were collected using a fluorescence microscope.

\section{RNA isolation and quantitative RT-PCR}

Total RNA was isolated using TRIzol reagent (Invitrogen). First-strand complementary DNA was synthesized using AMV-RTase (Promega), after which PCR was performed. Real-time $\mathrm{qPCR}$ reactions were performed in triplicate, and the final results were determined using a relative standard curve. The respective reverse transcription (RT)-PCR forward and reverse primer sequences were as follows: for rabaptin-5, 5'-TCATCATCCAGGAGGTTCAA- ${ }^{\prime}$ and 5' ${ }^{\prime}$-ACTCCCAGAAGGCTC TTTCA-3'; for GAPDH, 5'-GTGGTCTCCTCTGACTTCA AC-3' and 5'-TCTCTTCCTCTTGTGCTCTTG-3'; for PLD1, 5'-TGTCGTGATA CCACTTCTGCCA-3' and $5^{\prime}$-AGCATTTCGAGCTGCTGTTGAA-3'.

\section{ChIP assay}

Chromatin immunoprecipitation (ChIP) assay was performed as previously described, ${ }^{17}$ with minor modifications. The RABEP1 promoter region containing putative HIF- $1 \alpha$ binding sites was amplified by RT-PCR using the following primers: $5^{\prime}$-TTTTTT

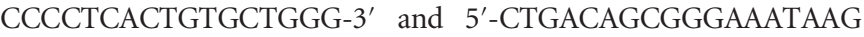
CAG-3'.

Establishments of transfected HT29 cell line and xenografts HT29 cells were transfected with GFP-PLD1-PH or empty vector. After transfection for $48 \mathrm{~h}$, cells were treated with $1000 \mu \mathrm{g} \mathrm{ml}{ }^{-1}$ of G418 for 30 days to select transfected cells, which were then used for xenografting. Female nude mice (5.5-weeks-old) were injected s.c. in the right flank with $4 \times 10^{6}$ HT29-GFP-PLD1-PH cells and in the left flank with $4 \times 10^{6}$ HT29-GFP-vector cells. Diameters of tumors in eight mice were measured three times weekly at right angles ( $a=$ short and $b=$ long) using electronic calipers, and tumor volumes were calculated by the formula volume $a \times b^{2} / 5$. Mice were killed 30 days after injection and split in half, after which one portion was utilized for immunohistochemistry and the other for biochemical analysis.

\section{Statistics}

The results are expressed as the mean \pm s.d. of the number of determinations indicated. Differences between groups were identified by analysis of variance, with a $P$-value $<0.05$ or 0.01 taken to indicate significance.

\section{RESULTS}

\section{PLD1 recovers HIF-1 $\alpha$-mediated inhibition of EGFR} endocytosis via the Rab5/7 pathway

To determine if PLD1 regulates the HIF- $1 \alpha$-induced inhibition of endocytosis, we investigated the expression of EGFR on ligand engagement. Ectopic expression of HIF-1 $\alpha$ prevented EGF $\left(100 \mathrm{ng} \mathrm{ml}^{-1}, 1 \mathrm{~h}\right)$ induced EGFR endocytosis, whereas overexpression of PLD1 promoted EGF-induced EGFR endocytosis and recovered endocytosis decelerated by HIF-1 $\alpha$ (Figure 1a). The effects of PLD1 on HIF-1 $\alpha$-mediated regulation of EGFR endocytosis were also assessed by rhodamine-labeled EGF clearance. HEK293 cells were treated with rhodamine-EGF and the internalized EGFR were visualized over time (Figure 1b). HIF-1 $\alpha$ reduced the amount of rhodamine-EGF uptake compared with that of the control. In contrast, PLD1 markedly increased clearance of rhodamineEGF and restored the rhodamine-EGF internalization inhibited by HIF-1 $\alpha$ (Figure 1b). Endocytosis, one of the deactivation pathways for receptors, involves a sequence of early and late 


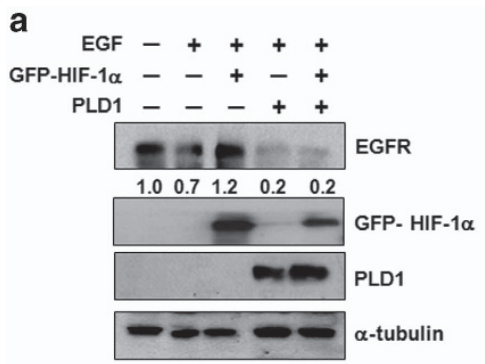

b
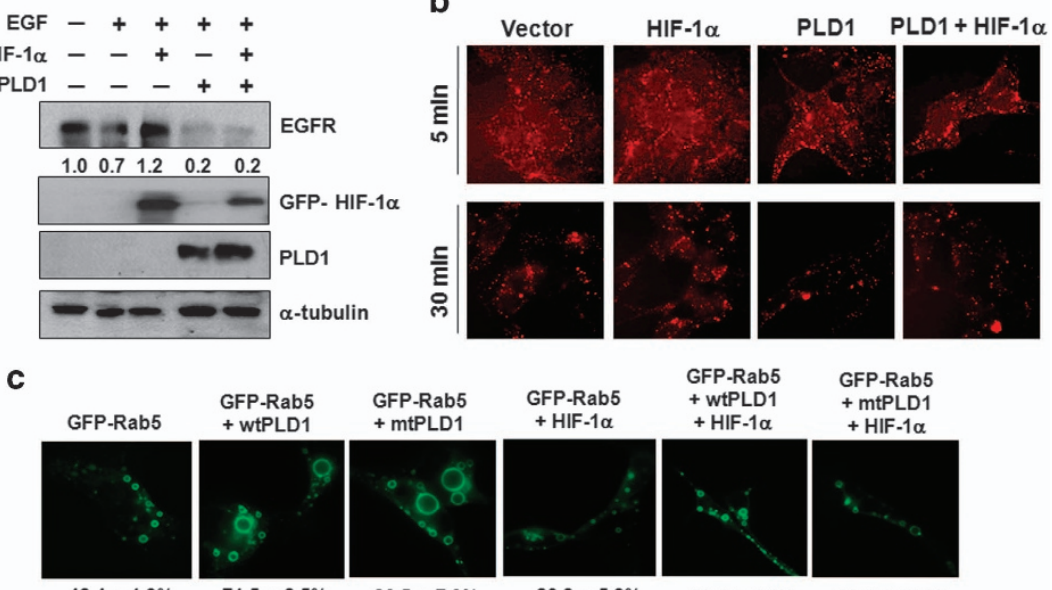

GFP-Rab5 GFP-Rab5

+ wtPLD1 + mtPLD

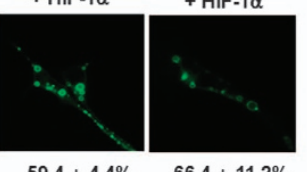

$42.4 \pm 1.9 \%$

$71.5 \pm 8.5 \%$

$80.5 \pm 7.9 \%$

$20.8 \pm 5.3 \%$

$59.4 \pm 4.4 \% \quad 66.4 \pm 11.2 \%$

Figure 1 PLD1 recovers HIF-1 $\alpha$-mediated inhibition of EGFR endocytosis via the Rab5/7 pathway. (a) HEK293 cells were transfected with GFP-HIF- $1 \alpha$ and PLD1, then incubated in serum-free media for $14 \mathrm{~h}$ and stimulated with EGF $\left(100 \mathrm{ng} \mathrm{ml}^{-1}\right)$ for $1 \mathrm{~h}$. The indicated proteins were analyzed by western blot. The values were normalized to that of $\alpha$-tubulin and expressed relative to the control. (b) After HEK293 cells were transfected with GFP-HIF-1 $\alpha$ and/or PLD1, they were treated with Alexa Fluor 555-EGF (20 ng ml-1) for the indicated times, fixed and examined by fluorescence microscopy. (c) HEK293 cells were transfected with GFP-Rab5 (Q79L), wtPLD1, mtPLD1 and $\mathrm{HA}-\mathrm{HIF}-1 \alpha$, then visualized by fluorescence microscopy. Data are representative of three independent experiments. EGFR, epidermal growth factor receptor; HA, hemagglutanin; HIF-1 $\alpha$, hypoxia-inducible factor- $1 \alpha$; PLD, phospholipase D.

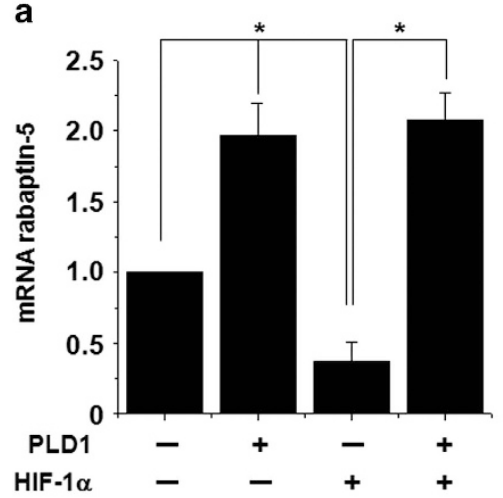

C

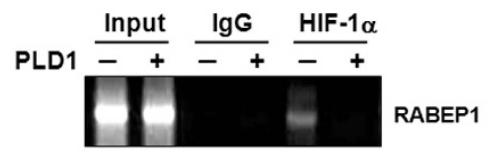

b
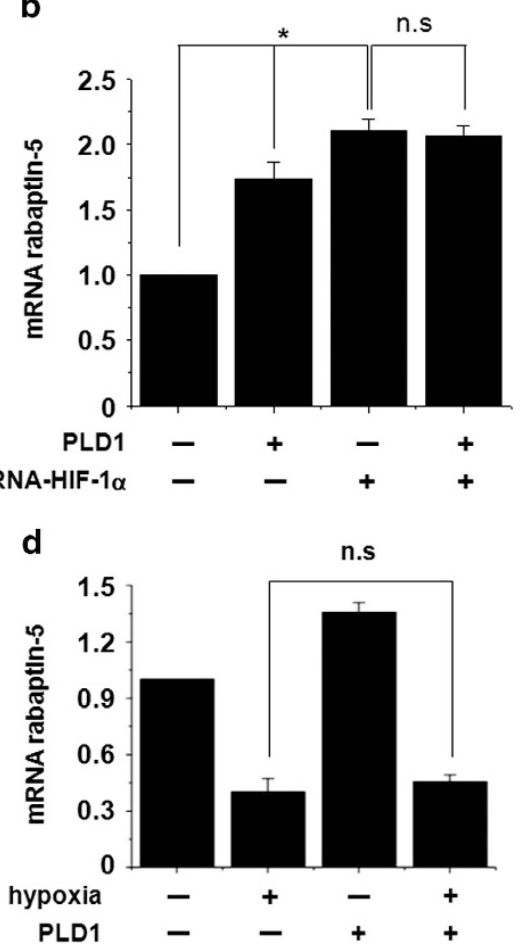

Figure 2 PLD1 regulates the expression of the Rab5 effector, rabaptin-5, through HIF-1 $\alpha$ in an oxygen-dependent manner. (a) Quantitative RT-PCR of rabaptin-5 mRNA in HEK293 cells transfected with the indicated constructs. ${ }^{*} P<0.05$. (b) Quantitative RT-PCR of rabaptin-5 mRNA in HEK293 cells transfected with PLD1 or siRNA for HIF-1 $\alpha .{ }^{*} P<0.05$. (c) ChIP assay of the binding of HIF-1 $\alpha$ to the RABEP1 promoter in HEK293 cells transfected with PLD1. (d) Quantitative RT-PCR assay of rabaptin-5 mRNA in HEK293 cells transfected with the indicated constructs under hypoxia or normoxia. Results are shown as the mean \pm s.d. of the three independent experiments. EGFR, epidermal growth factor receptor; HIF-1 $\alpha$, hypoxia-inducible factor- $1 \alpha$; ChIP, chromatin immunoprecipitation; GFP, green fluorescent protein; NS, non-significant; PLD, phospholipase D; RT-PCR, reverse transcription-PCR. 
endosomal fusion events orchestrated by Rabs, a group of Ras-like small GTPases, ${ }^{18}$ that ultimately leads to degradation of internalized receptors in lysosomes. As an activator of early endosome fusion, Rab5 has a role in early endosome docking and fusion, and the expression of constitutively active Rab5 (Q79L) that is preferentially in the GTP-bound state results in the formation of giant early endosomes. ${ }^{19}$ To determine if EGFR internalization was influenced by the endocytic cycle, we transfected HEK293 cells with constitutively active GFP-Rab5 (Q79L), an early endosome marker, and then checked the formation of endosomes. Wild-type (wt) PLD1 or lipaseinactive mutant (mt) PLD1-transfected cells showed giant early endosomes, consistent with the enhanced membrane fusion that results from active Rab5 ${ }^{19}$ (Figure 1c). The endosome fusion was retarded by HIF- $1 \alpha$, and HIF- $1 \alpha$-dependent attenuation of endosome fusion was recovered by wtPLD1 or mtPLD1 (Figure 1c). The fluorescence recovery of GFP-Rab7, a late endosome marker, on vesicles was also comparable to that of GFP-Rab5 (data not shown). These finding demonstrate that PLD1 increases Rab5/Rab7-mediated endosome fusion independent of lipase activity and recovers endosome fusion decelerated by HIF- $1 \alpha$.

\section{PLD1 regulates the expression of the Rab5 effector,} rabaptin-5, through HIF- $1 \alpha$ in an oxygen-dependent manner Rabaptin-5 contains two Rab5-binding sites required for binding Rab5 in early endosomes in a GTP-dependent manner, and immunodepletion of rabaptin-5 strongly inhibits Rab5dependent early endosome fusion. ${ }^{20,21}$ Thus, rabaptin-5 is considered a critical effector of Rab5. Since HIF-1 $\alpha$ was reported to attenuate Rab5-mediated early endosome fusion via downregulation of rabaptin5, we examined whether PLD1 affects expression of RABEP1 (rabaptin-5) RNA expression levels. Ectopic expression of PLD1 significantly increased a

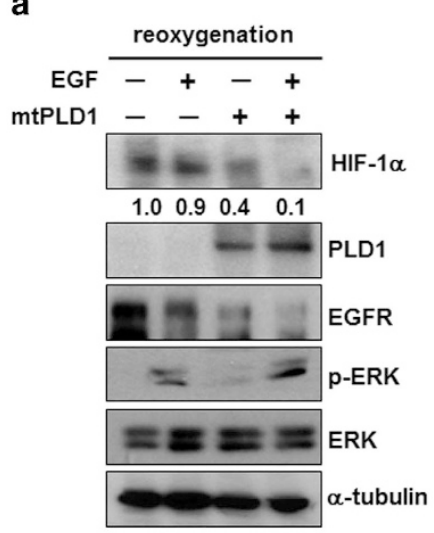

d

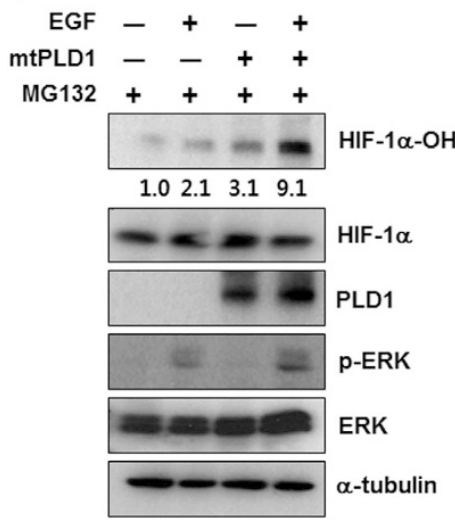

b
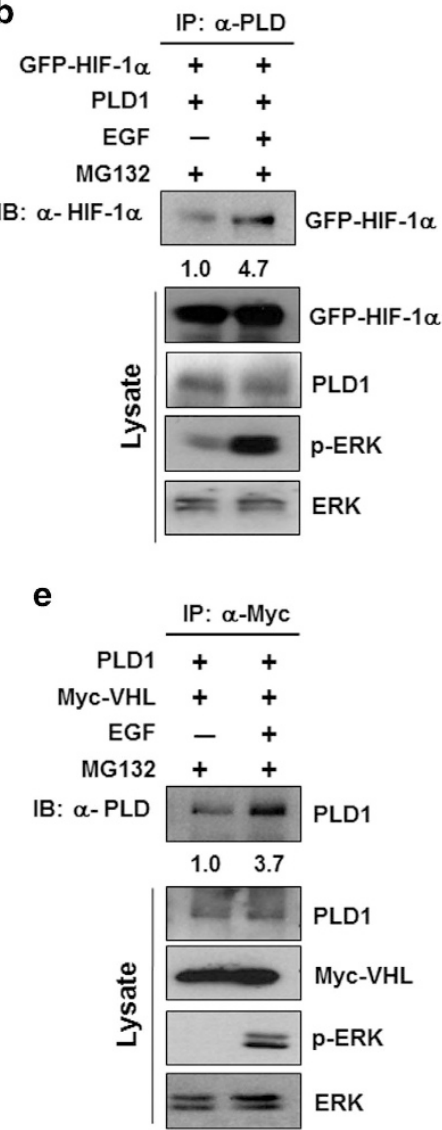

C

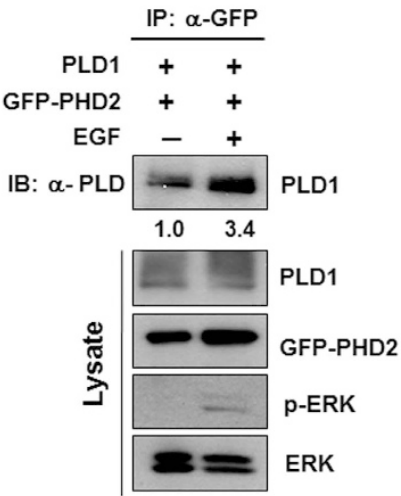

Figure 3 PLD1 promotes EGFR endocytosis by destabilizing HIF-1 $\alpha$ protein via the PHD2/VHL-mediated pathway. (a) HEK293 cells were transfected with mtPLD1, then incubated under hypoxia. After hypoxia for $4 \mathrm{~h}$, the cells were reoxygenized and treated with EGF $\left(100 \mathrm{ng} \mathrm{ml}^{-1}\right)$ for $30 \mathrm{~min}$. The lysates were then analyzed by immunoblot. The values were normalized to that of $\alpha$-tubulin and expressed as a fold of the control. ( $b$ and $\mathbf{c}$ ) HEK293 cells cotransfected with the indicated constructs and stimulated with EGF (100 ng ml-1) for $30 \mathrm{~min}$ in the presence MG132. The lysates were immunoprecipitated with anti-PLD (b) or anti-GFP (c) and immunoblotted with the indicated antibodies. The values were normalized against that of $\alpha$-tubulin and expressed as a fold of the control. (d) Immunoblot assay of the indicated proteins in HEK293 cells transfected with mt PLD1 and stimulated with EGF in the presence MG132. The hydroxylated values were normalized to total HIF-1 $\alpha$ and expressed as a fold of the control. (e) HEK293 cells transfected with mt PLD1 and stimulated with EGF in the presence MG132. The lysates were immunoprecipitated and immunoblotted with the indicated antibodies. Data are representative of three independent experiments. EGFR, epidermal growth factor receptor; HIF-1 $\alpha$, hypoxia-inducible factor-1 $\alpha$; GFP, green fluorescent protein; PLD, phospholipase D; VHL, von Hippel-Lindau. 


\section{a}

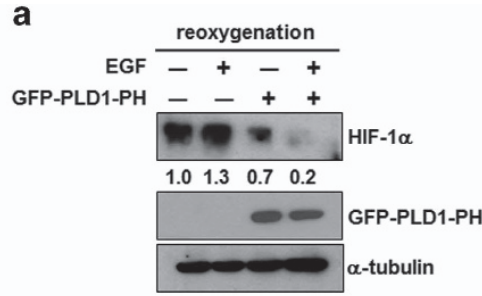

b
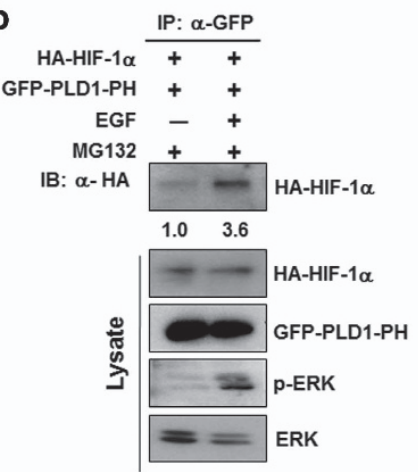

e

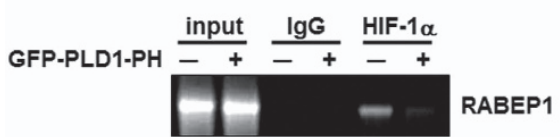

C

d
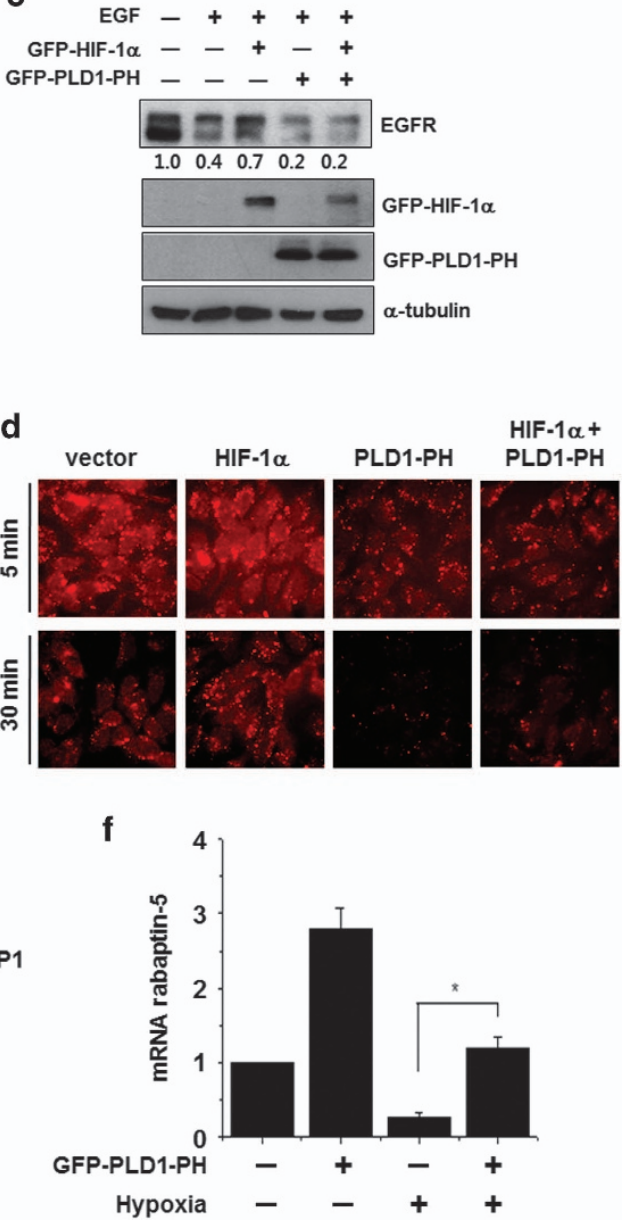

Figure 4 PH-domain of PLD1 is crucial for EGFR endocytosis by increasing the level of rabaptin-5. (a) HEK293 cells transfected with GFP-PLD1-PH and incubated under hypoxia. After hypoxia for $4 \mathrm{~h}$, the cells were reoxygenized with treatment of EGF (100 ng ml-1) for $30 \mathrm{~min}$. The values were normalized to that of $\alpha$-tubulin and expressed as a fold of the control. (b) HEK293 cells were cotransfected with the indicated constructs, then exposed to EGF $\left(100 \mathrm{ng} \mathrm{ml}^{-1}\right)$ for $30 \mathrm{~min}$ in the presence of MG132. The lysates were immunoprecipitated and immunoblotted with the indicated antibodies. (c) Immunoblot assay of EGFR under the indicated condition. The values were normalized to that of $\alpha$-tubulin and expressed as a fold of the control. (d) HEK293 cells were cotransfected with GFP-HIF-1 $\alpha$ and GFP-PLD1-PH. After $24 \mathrm{~h}$ of serum starvation, the cells were treated with Alexa Fluor 555-EGF $\left(20 \mathrm{ng} \mathrm{ml}^{-1}\right)$ for the indicated times, fixed and examined by fluorescence microscopy. Data are representative of three independent experiments. (e) ChIP assay for the binding of HIF- $1 \alpha$ to the RABEP1 promoter in HEK293 cells transfected with PLD1-PH. (f) Quantitative RT-PCR assay of rabaptin-5 mRNA in HEK293 cells transfected with GFP-PLD1-PH and incubated under hypoxia. ${ }^{*} P<0.05$, results are shown as the mean \pm s.d. of the three independent experiments. EGFR, epidermal growth factor receptor; HIF-1 $\alpha$, hypoxia-inducible factor-1 $\alpha$; GFP, green fluorescent protein; $\mathrm{PH}$, pleckstrin homology; PLD, phospholipase D.

RABEP1 RNA expression and recovered HIF- $1 \alpha$-mediated downregulation of rabaptin-5 (Figure 2a). Moreover, knockdown of HIF-1 $\alpha$ increased the mRNA level of RABEP1, while PLD1 did not affect rabaptin-5 expression in HIF-1 $\alpha$ depleted cells, suggesting that PLD1 might affect expression of rabaptin-5 through HIF-1 $\alpha$ (Figure $2 \mathrm{~b}$ ). Since RABEP1 promoters are known to contain a conserved hypoxia responsive element, we further examined whether PLD1 influences the binding of HIF- $1 \alpha$ to promoter of RABEP1 using a ChIP assay. PLD1 dramatically suppressed the binding of HIF- $1 \alpha$ to the promoter of the RABEP1, which subsequently promoted expression of rabaptin-5 (Figure 2c). Further, PLD1 did not recover the level of $R A B E P 1$ decreased by hypoxia (Figure $2 \mathrm{~d}$ ).
These data suggest that PLD1 modulates rabaptin-5 expression through HIF- $1 \alpha$ in an oxygen-dependent manner.

PLD1 promotes EGFR endocytosis by destabilizing HIF-1 $\alpha$ protein via a PHD2/VHL-mediated pathway

We recently demonstrated that PLD1 protein itself destabilizes HIF- $1 \alpha$ protein by interacting directly with the components involved in VHL-dependent degradation of HIF-1 $\alpha$, independent of PLD activity. ${ }^{15}$ Our results show that PLD1 and HIF- $1 \alpha$ have opposite roles in endocytosis. We examined whether PLD1-mediated HIF-1 $\alpha$ degradation occurs during EGFR endocytosis. As a control, lipase inactive mtPLD1 decreased the level of HIF-1 $\alpha$ and EGF promoted mtPLD1-mediated 
a
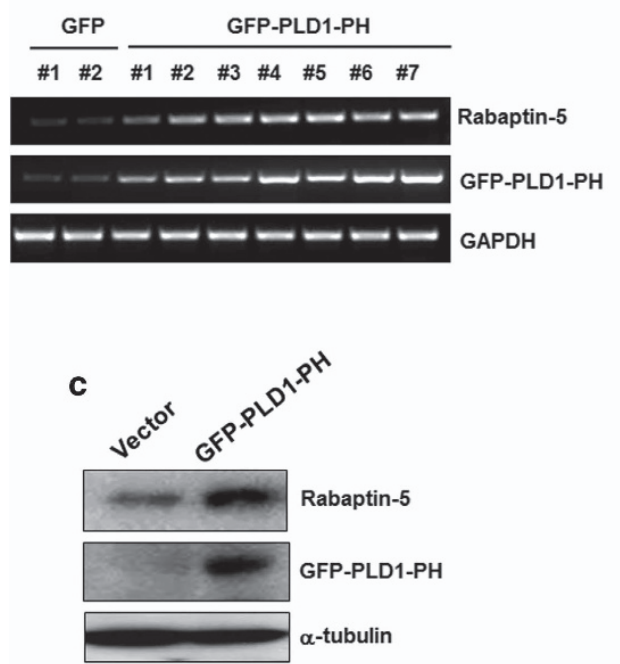

b

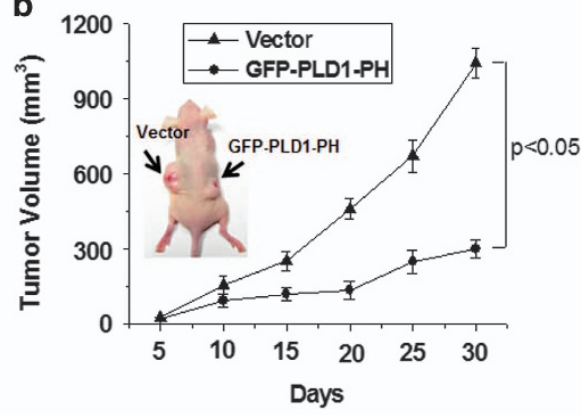

d

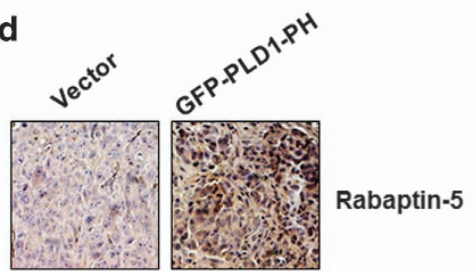

Figure 5 PLD1-PH attenuates tumor progression. (a) RT-PCR assay of rabaptin-5 mRNA in HT29 cells stably transfected with PLD1-PH. (b) HT29 cells expressing empty vector or PLD1-PH cells were xenografted on the left and right flanks of nude mice, respectively (arrows), then analyzed for tumor volume. Photographs show the representative tumors. Data were expressed as the mean $\pm \mathrm{s}$.d. of seven different mice. (c) Immunoblot analysis of rabaptin-5 in tumor tissues from the xenografted mice injected with vector and PLD1-PH-expressed HT29 cells. Data are representative of three independent experiments. (d) Immunohistochemical staining of rabaptin-5 in the tumor tissues of xenografted mice injected with vector and PLD1-PH-expressed HT29 cells. Data are representative of three independent experiments. $\mathrm{PH}$, pleckstrin homology; PLD, phospholipase D; RT-PCR, reverse transcription-PCR.

HIF-1 $\alpha$ degradation (Figure 3a). Moreover, EGF increased mtPLD1-induced EGFR endocytosis (Figure 3a). PLD1 protein itself acts as a molecular platform, associating directly with HIF-1 $\alpha$, PHD2 and VHL, thereby assembling a multi-protein complex that mediates efficient degradation of HIF-1 $\alpha .{ }^{15}$ Thus, we examined whether EGF affects their interaction. EGF enhanced interaction of PLD1 with HIF-1 $\alpha$ and PHD2 (Figures $3 \mathrm{~b}$ and $\mathrm{c}$ ). PLD1 increases prolyl hydroxylation of HIF- $1 \alpha$ via interaction with PHD2, followed by degradation of HIF-1 $\alpha .{ }^{15}$ EGF promoted PLD1-induced hydroxylation of HIF-1 $\alpha$ (Figure 3d). Furthermore, EGF increased interaction of PLD1 with VHL (Figure 3e). Taken together, findings suggest that PLD1 may promote EGFR endocytosis by destabilizing HIF-1 $\alpha$ via the PHD2/VHL-mediated pathway.

\section{PH-domain of PLD1 is crucial for EGFR endocytosis by increasing the level of rabaptin-5}

PA, a product of PLD activation, is important for receptormediated endocytosis. ${ }^{22,23}$ The PX domain of PLD1 itself accelerates EGFR endocytosis dependent on the GAP activity of dynamin, not on the activity of PLD. ${ }^{9}$ We recently reported that the PH domain of PLD1 (PLD1-PH) is required for degradation of HIF- $1 \alpha$ via direct interaction with HIF-1 $\alpha$, PHD2 and VHL. ${ }^{15}$ Since PLD1 destabilizes HIF- $1 \alpha$ and increases EGFR endocytosis, we investigated the possibility that PLD1-PH is responsible for EGFR endocytosis. EGF increased PLD1-PH-mediated destabilization of HIF- $1 \alpha$ and interaction of PLD1-PH with HIF-1 $\alpha$ (Figures $4 \mathrm{a}$ and $\mathrm{b}$ ). Moreover, PLD1-PH recovered HIF- $1 \alpha$-mediated suppression of EGFR endocytosis on EGF treatment (Figure 4c). The HEK293 cells were stimulated with rhodamine-EGF and internalized EGFR was visualized over time (Figure $4 \mathrm{~d}$ ). In contrast to HIF-1 $\alpha$, which reduced the amount of rhodamine-EGF uptake, PLD1-PH markedly increased clearance of rhodamine-EGF and restored rhodamine-EGF internalization suppressed by HIF- $1 \alpha$ relative to the control (Figure $1 \mathrm{~b}$ ). Thus, these findings suggest that PLD1-PH itself can promote EGFR endocytosis and recover endocytosis attenuated by HIF-1 $\alpha$. To further examine whether PLD1-PH increases endocytosis via regulation of rabaptin-5 expression, we examined if PLD1-PH affects the binding of HIF-1 $\alpha$ to promoter of RABEP1 using a ChIP assay. Interestingly, PLD1-PH dramatically abolished the recruitment of HIF-1 $\alpha$ onto the promoter of $R A B E P 1$ (Figure 4e). Moreover, PLD1-PH recovered the level of $R A B E P 1$ inhibited by hypoxia (Figure $4 \mathrm{f}$ ), contrary to intact PLD1, which did not recover the level of RABEP1 suppressed under hypoxia (Figure 2d). Taken together, these results suggest that the PH domain of PLD1 itself destabilizes HIF-1 $\alpha$, increases the level of rabaptin-5 and accelerates EGFR endocytosis.

\section{PLD1-PH attenuates tumor progression}

Tumor hypoxia or oncogenic activation of HIF- $1 \alpha$ lengthens RTK-mediated signaling by delaying endocytosis-mediated deactivation of receptors. ${ }^{13}$ Since the EGF-engaged EGFR turnover rate is shortened, in part owing to PLD1-PHmediated acceleration of the endocytic cycle, we examined the effect of PLD-PH on tumor growth. Various clones of HT29 colorectal cancer cells overexpressing PLD1-PH showed increased expression of rabaptin-5 compared with that of 
control vector (Figure 5a). Stable HT29 cells expressing vector or PLD1-PH were injected into mice to generate xenografted tumors. PLD1-PH-expressed HT29 showed significantly decreased tumor growth relative to control cells (Figure 5b). Immunoblot and immune staining revealed that the levels of rabaptin- 5 were markedly increased in tumor tissues derived from HT29-PLD1-PH tumors (Figures $5 c$ and d). Thus, it is suggested that PLD1-PH attenuates tumorigenesis, probably via induction of rabaptin-5 expression, which promotes endocytic cycling.

\section{DISCUSSION}

In the present study, we demonstrated that PLD1 recovers the EGFR endocytosis decelerated by HIF- $1 \alpha$ independent of lipase activity via the Rab5-mediated endosome fusion pathway. Moreover, we showed that the PH domain of PLD1 itself has a novel role in EGFR endocytosis by increasing the expression of rabaptin-5, a Rab5 effector downregulated by HIF- $1 \alpha$. During receptor-mediated endocytosis, PA is involved in the membrane curvature required for vesicle budding. ${ }^{8}$ The GAP property of PLD for dynamin is also required for EGFRinduced endocytosis. ${ }^{9}$ Dynamin is a large GTP-binding protein that mediates endocytosis by hydrolyzing GTP. ${ }^{24}$ It has been suggested that, depending on EGF concentration, PLD is involved in EGFR endocytosis through both the GAP activity of its PX domain for dynamin and the production of PA. ${ }^{9}$ RTKs are central components of cell signaling networks that have crucial roles in normal and pathological processes, including cancer. ${ }^{25}$ Hypoxia is associated with enhanced RTK-mediated signaling, which contributes to oncogenesis and more aggressive disease. ${ }^{26-28}$ It has been proposed that HIF- $1 \alpha$ upregulates signaling of EGFR and other RTK by decelerating clathrin-mediated endocytosis at the early endosome sorting stage, resulting in enhanced cell proliferation and survival. ${ }^{13}$ Furthermore, HIF promotes the formation of caveolae via positive regulation of caveolin-1, resulting in tumor cell proliferation. ${ }^{29}$ Moreover, HIF-1 decreases the degradation of ligand-engaged EGFR by attenuating Rab5mediated early endosome fusion via downregulation of rabaptin-5. ${ }^{13}$ The lengthened EGFR turnover extends EGFRmediated signaling; however, it is unknown whether PLD affects Rab5-mediated early endosome fusion via modulation of HIF-1. We recently reported that PLD1 or PLD1-PH domain acts as a novel HIF- $1 \alpha$ regulator, functioning as a microenvironment for efficient post-translational modifications of HIF-1 $\alpha$, leading to degradation of the protein. ${ }^{15}$ Here, we show that PLD1-PH promotes EGFR endocytosis by downregulating HIF- $1 \alpha$ and increasing rabaptin-5. Despite functional similarities in the tumorigenesis, HIF-1 $\alpha$ and PLD1 are believed to have distinct roles in endocytosis. Unlike HIF-1 $\alpha$, PLD1 positively regulates clathrin-mediated endocytosis via facilitation of membrane traffic $c^{30,31}$. PLD activity is implicated in endocytosis of transferrin receptor, EGFR and angiotensin II receptor. 22,23,32 EGFs cause conformational changes in PLD1, after which the PH domain of PLD1 binds $\mu 2$, a subunit of adaptor protein 2 (AP2), which facilitates recruitment of endocytic components. ${ }^{33}$ Thus, it is suggested that EGFR endocytosis is coordinated by HIF- $1 \alpha$ and PLD1. Ligand-engaged EGFR turnover is prolonged as a result of HIF-dependent transcriptional repression of rabaptin-5, and the lengthened existence of internalized EGFR increases EGFR signaling to promote proliferation and cell survival. ${ }^{13}$ Although receptor endocytosis is a major negative feedback loop, the internalized receptors remain active while en route for recycling or degradation. Although it is currently unclear how the endocytic program influences quantitative aspects of output signals, the phenotypes of cells that have defective components of the endocytic machinery clearly indicate strong relationships between receptor endocytosis and restrained signaling outcomes. ${ }^{34}$ The PH domain of PLD appears to act as a center that forms multicomplexes with binding partners. These dynamics of PLD-PH might regulate EGF signaling and determine signaling duration and strength.

PLD1-PH, unlike intact PLD1, decreases the stability of HIF- $1 \alpha$ in both normoxia and hypoxia. ${ }^{15}$ Here, we demonstrate that, contrary to intact PLD1, PLD1-PH recovers the expression of rabaptin-5 decreased under hypoxia. Moreover, PLD1-PH itself suppresses tumor progression, but increases the expression of rabaptin- 5 decreased by HIF- $1 \alpha$. In addition, PLD1-PH abolishes the expression of HIF- $1 \alpha$ and its target genes under normoxia and hypoxia. ${ }^{15}$ Therefore, modulation of EGFR endocytosis by PLD1-PH may benefit cancer therapeutic intervention.

\section{CONFLICT OF INTEREST}

The authors declare no conflict of interest.

\section{ACKNOWLEDGEMENTS}

This study was supported by a National Research Foundation of Korea (NRF) grant funded by the Korean government (NRF-2015R1A2A1A05001884) and a Translational Research Center for Protein Function Control Grant (NSF 2009-0092960) and a National R\&D Program for Cancer Control grant from the Ministry for Health, Welfare, and Family Affairs (Republic of Korea; 0920050).

1 Sorkin $A$, von Zastrow M. Endocytosis and signalling: intertwining molecular networks. Mol Cell Biol 2009; 10: 609-622.

2 Lee CS, Kim KL, Jang JH, Choi YS, Suh PG, Ryu SH. The roles of phospholipase D in EGFR signaling. Biochim Biophys Acta 2009; 1791: 862-868.

3 Kang DW, Choi CY, Cho YH, Tian H, Di Paolo G, Choi KY et al. Targeting phospholipase D1 attenuates intestinal tumorigenesis by controlling $\beta$-catenin signaling in cancer-initiating cells. J Exp Med 2015; 212: 1219-1237.

4 Hwang WC, Kim MK, Song JH, Choi KY, Min DS. Inhibition of phospholipase D2 induces autophagy in colorectal cancer cells. Exp Mol Med 2014; 46: e124.

5 Jang YH, Choi KY, Min DS. Phospholipase D-mediated autophagic regulation is a potential target for cancer therapy. Cell Death Differ 2014; 21: 533-546.

6 Kang DW, Choi K, Min DS. Functional regulation of phospholipase D expression in cancer and inflammation. J Biol Chem 2014; 289: 22575-22582. 
7 Jang JH, Lee CS, Hwang D, Ryu SH. Understanding of the roles of phospholipase $\mathrm{D}$ and phosphatidic acid through their binding partners. Prog Lipid Res 2012; 51: 71-81.

8 Jenkins GM, Frohman MA. Phospholipase D: a lipid centric review. Cell Mol Life Sci 2005; 62: 2305-2316.

9 Lee CS, Kim IS, Park JB, Lee MN, Lee HY, Suh PG et al. The phox homology domain of phospholipase D activates dynamin GTPase activity and accelerates EGFR endocytosis. Nat Cell Biol 2006; 8: 477-484.

10 Stenmark H, Parton RG, Steele-Mortimer O, Lütcke A, Gruenberg J, Zerial M. Inhibition of rab5 GTPase activity stimulates membrane fusion in endocytosis. EMBO J 1994; 13: 1287.

11 Harris AL. Hypoxia-a key regulatory factor in tumour growth. Nat Rev Cancer 2002; 2: 38-47.

12 Semenza GL. Targeting HIF-1 for cancer therapy. Nat Rev Cancer 2003; 3 : 721-732.

13 Wang Y, Roche O, Yan MS, Finak G, Evans AJ, Metcalf JL et al. Regulation of endocytosis via the oxygen-sensing pathway. Nat Med 2009; 15: 319-324.

14 Zhong H, De Marzo AM, Laughner E, Lim M, Hilton DA, Zagzag D et al. Overexpression of hypoxia-inducible factor lalpha in common human cancers and their metastase. Cancer Res 1999; 59: 5830-5835.

15 Park MH, Choi KY, Jung Y, Min DS. Phospholipase D1 protein coordinates dynamic assembly of HIF- $1 \alpha-P H D-V H L$ to regulate HIF- $1 \alpha$ stability. Oncotarget 2014; 5: 11857-11872.

16 Min DS, Ahn BH, Rhie DJ, Yoon SH, Hahn SJ, Kim MS et al. Expression and regulation of phospholipase $\mathrm{D}$ during neuronal differentiation of PC12 cells. Neuropharmacol 2001; 41: 384-391.

17 Park MH, Ahn BH, Hong YK, Min DS. Overexpression of phospholipase D enhances matrix metalloproteinase-2 expression and glioma cell invasion via protein kinase $\mathrm{C}$ and protein kinase A/NF-kappaB/Sp1-mediated signaling pathways. Carcinogenesis 2009; 30: 356-365.

18 Pfeffer SR. Motivating endosome motility. Nat Cell Biol 1999; 1: E145-E147.

19 Bucci C, Parton RG, Mather IH, Stunnenberg H, Simons K, Hoflack B et al. The small GTPase rab5 functions as regulatory factor in the early endocytic pathway. Cell 1992; 70: 729-740.

20 Korobko EV, Palgova IV, Kiselev SL, Korobko IV. Apoptotic cleavage of rabaptin-5-like proteins and a model for rabaptin-5 inactivation in apoptosis. Cell Cycle 2006; 5: 1854-1858.

21 Stenmark H, Vitale G, Ullrich 0, Zerial M. Rabaptin-5 is a direct effector of the small GTPase Rab5 in endocytic membrane fusion. Cell 1995; 83 : 423-432.

22 Du G, Huang P, Liang BT, Frohman MA. Phospholipase D2 localizes to the plasma membrane and regulates angiotensin II receptor endocytosis. Mol Biol Cell 2004; 15: 1024-1030.

23 Shen Y, Xu L, Foster DA. Role for phospholipase D in receptor-mediated endocytosis. Mol Cell Biol 2001; 21: 595-602.
24 Henley JR, Krueger EW, Oswald BJ, McNiven MA. Dynamin-mediated internalization of caveolae. J Cell Biol 1998; 141: 85-99.

25 Blume-Jensen P, Hunter T. Oncogenic kinase signalling. Nature 2001; 411: 355-365.

26 Franovic A, Gunaratnam L, Smith KRI, Patten D, Lee S. Translational up-regulation of the EGFR by tumor hypoxia provides a nonmutational explanation for its overexpression in human cancer. Proc Natl Acad Sci USA 2007; 104: 13092-13097.

27 Pennacchietti S, Michieli P, Galluzzo M, Mazzone M, Giordano S, Comoglio PM. Hypoxia promotes invasive growth by transcriptional activation of the met protooncogene. Cancer Cell 2003; 3: 347-361.

28 Koochekpour S, Jeffers M, Wang PH, Gong C, Taylor GA, Roessler LM et al. The von Hippel-Lindau tumor suppressor gene inhibits hepatocyte growth factor/scatter factor-induced invasion and branching morphogenesis in renal carcinoma cells. Mol Cell Biol 1999; 19: 5902-5912.

29 Wang Y, Roche O, Xu C, Moriyama EH, Heir P, Chung J et al. Hypoxia promotes ligand-independent EGF receptor signaling via hypoxia-inducible factor-mediated upregulation of caveolin-1. Proc Natl Acad Sci USA 2012; 109: 4892-4897.

30 Donaldson JG. Phospholipase $D$ in endocytosis and endosomal recycling pathways. Biochim Biophys Acta 2009; 1797: 845-849.

31 Selvy PE, Lavieri RR, Lindsley CW, Brown HA. Phospholipase d: enzymology, functionality, and chemical modulation. Chem Rev 2011; 111: 6064-6119.

32 Botelho RJ, Teruel M, Dierckman R, Anderson R, Wells A, York JD et al. Localized biphasic changes in phosphatidylinositol-4,5-bisphosphate at sites of phagocytosis. J Cell Biol 2000; 151: 1353-1368.

33 Lee JS, Kim IS, Kim JH, Cho W, Suh PG, Ryu SH. Determination of EGFR endocytosis kinetic by auto-regulatory association of PLD1 with mu2. PLoS One 2009; 4: 7090.

34 Avraham R, Yarden Y. Feedback regulation of EGFR signalingLdecision making by early and delayed loops. Nat Rev Mol Cell Biol 2011; 12: 104-117.

(c) (i) (2) (2) This work is licensed under a Creative Commons Attribution-NonCommercial-ShareAlike 4.0 International License. The images or other third party material in this article are included in the article's Creative Commons license, unless indicated otherwise in the credit line; if the material is not included under the Creative Commons license, users will need to obtain permission from the license holder to reproduce the material. To view a copy of this license, visit http://creativecommons.org/licenses/by-nc-sa/4.0/ 